Archived version from NCDOCKS Institutional Repository http://libres.uncg.edu/ir/asu/

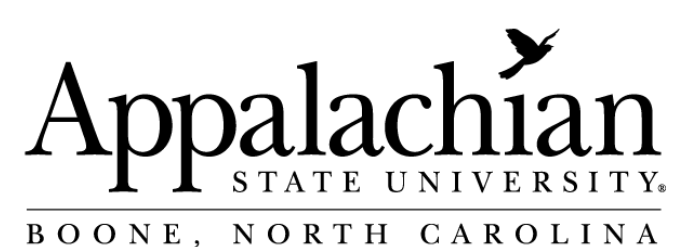

\title{
Using Rspec In An Introductory Bright Star Spectroscopy Lab Activity
}

\author{
By: James Howe and David J. Sitar
}

\begin{abstract}
The premise of this activity (developed under the Smoky Mountain STEM Initiative Research Grant) is that introductory astronomy students who participate in every aspect of spectroscopic analysis-from image capture to calibrated, curve-divided creation of graphs - will have a deeper under- standing of the fundamental tools of spectroscopy. Being in- volved with an activity, completing it, and sharing results are active aids to learning.
\end{abstract}

Howe, J. and D. J. Sitar (2017). "Using RSpec in an introductory bright star spectroscopy lab activity." The Physics Teacher 56(1): 56-57. doi: 10.1119/1.5018698. Publisher version of record available at: https://aapt.scitation.org/ doi/10.1119/1.5018698 


\section{Using RSpec in an introductory bright star spectroscopy lab activity}

James Howe, Southwestern Community College, Sylva, NC 28779; David J. Sitar, Appalachian State University, Boone, NC 28608; sitardj@appstate.edu

\begin{abstract}
$\Delta$ fter presenting at the North Carolina Section of the American Association of Physics Teachers during the fall 2016 meeting, we were encouraged to turn our poster into a paper. This article describes the strengthening of a bright star spectroscopy lab activity for introductory astronomy lab students (AST1002) at Appalachian State University. Explanations of the tools and methods used in the activity are included, particularly the preparation of additional materials using RSpec and calibrated instrument response curves.
\end{abstract}

\section{Motivation}

The premise of this activity (developed under the Smoky Mountain STEM Initiative Research Grant) is that introductory astronomy students who participate in every aspect of spectroscopic analysis-from image capture to calibrated, curve-divided creation of graphs-will have a deeper understanding of the fundamental tools of spectroscopy. Being involved with an activity, completing it, and sharing results are active aids to learning.

\section{Hardware and software}

The software RSpec was used in this activity to engage students in imaging spectra and analyzing chemical compositions of bright stars. An SBIG STF8300C CCD camera, a diffraction grating with 600 lines $/ \mathrm{mm}$, and an 11-inch Celestron Cassegrain telescope were used to obtain images of bright stars alongside their spectra. To produce more accurate and useful spectra, a precise instrument response curve had to be created for our equipment, which proved challenging due to the current diffraction grating and distance from grating to CCD chip. However, the lab activity does function in its present state as a vivid and appealing introduction to spectroscopy.

Images were processed in Nebulosity and then analyzed in RSpec using the software's calibration of Vega, an A0 star. This project has encouraged us to dig deeper into RSpec's features and has ultimately increased our understanding of image processing with spectral data analysis, a laboratory exercise we had not been able to do in the past at our institution.

\section{Previous student activity}

Past students had not had the opportunity to do spectroscopy and spectral analysis in our introductory astronomy lab (AST1002) on this level. However, now (thanks to some funding) our new cameras and diffraction gratings pair well in creating spectral images. With the older setup, students were able to image small segments of spectra, but never did any analysis (see Fig. 1). Images like Fig. 1 were taken with an SBIG STV camera with no software available to reduce spectra easily. Therefore, what students saw were just the spectra opened in Astroart; the vertical black lines are the absorption lines, the "UPC code," if you will, of what the star is made of. One could argue that this is still "pretty neat," but we argue that it is not pedagogically sound and that students are not getting the experience needed to understand spectroscopy.

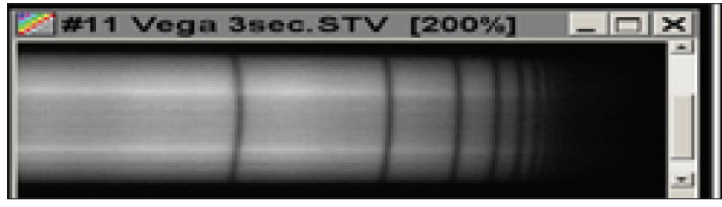

Fig. 1. Spectrum of star Vega.

\section{A new student activity}

Much brainstorming went into how we wanted to execute the activity. For students, the goal is to image different stars and determine star type and composition. Students will already be familiar with Nebulosity and have experience processing images based on past lab activities. By adding RSpec, they will now develop a hands-on understanding of spectroscopy and star classification. In addition, students will be able to view their curves in color fill, which provides an opportunity to make the connection between wavelength, frequency, and color.

\section{Calibration and results}

We created our instrument response curve from the data obtained (Fig. 2), which is an example of what the students see during processing. Figure 3 is our calibration and has a resolution of $2.9 \AA$ A per pixel.

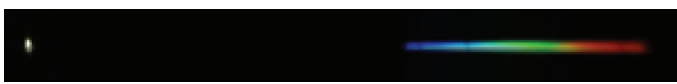

Fig. 2. Spectrum of Vega.

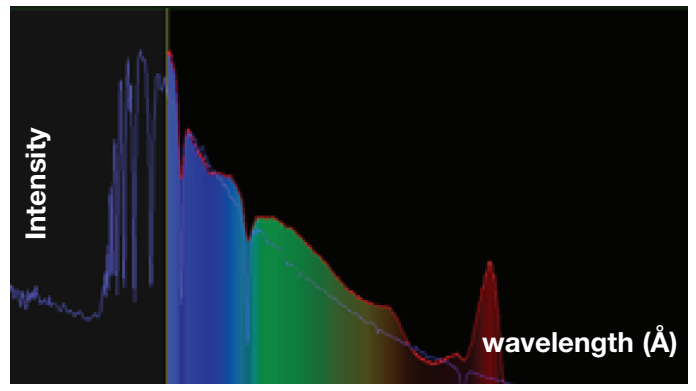

Fig. 3. Calibrated Vega compared to library Vega (blue line). 
With our current grating and spacing, the images captured were below the recommended resolution of 10-20 Å per pixel; therefore, an instrument response curve was difficult to calibrate. Once we calibrated, the $2.9 \AA$ per pixel instrument response curve was used for the rest of our data analysis. Even at suboptimal resolution, a worthwhile instrument response curve can be created and used to analyze spectra of other stars. Students should now be able to calibrate, using these parameters, and identify types and compositions of stars based on the built-in RSpec reference library. Figure 4 is another one of our examples; however, for reasons we cannot yet explain there is a slight shift to the right of our data compared to the reference line (in blue), but we were still extremely happy with our first light results. The reason red is so dominant in Fig. 4 is because Aldebaran is an older, redder star; therefore, it appears shifted red in the spectrum.

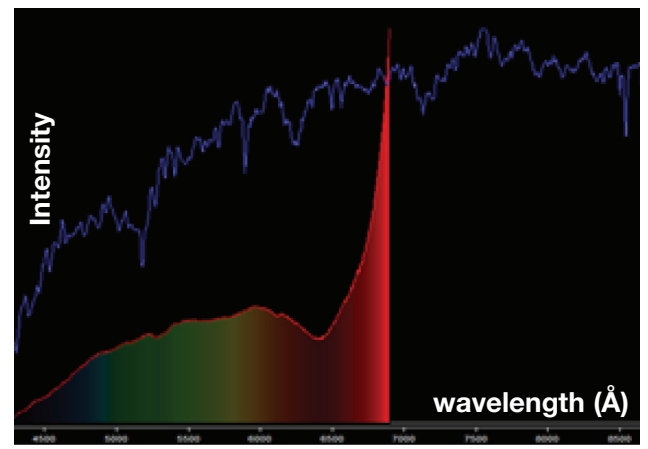

Fig. 4. Calibrated Aldebaran compared to library Aldebaran (blue line).

Next is an example of how a final spectral analysis should appear. Notice in Fig. 5 that Sirius' spectral curve has been superimposed with chemical composition lines to determine star type. Notice our data, the red curved dips $(\mathrm{Ha}, \mathrm{H} \beta$, and $\mathrm{H} \gamma$ ), superbly align with each of the blue library reference lines. These lines also coincide with the strongest absorption lines from Fig. 6. Students will be expected to find similar results for other bright stars. We should also point out that the large singular peak at zero in Fig. 5 is the light from the star itself. Figure 6 has been zoomed in to show absorption lines, but if the figure showed the whole image, you would also see the star. Refer back to Fig. 2 to see an example of the complete image.

\section{Conclusion and future of project}

We have confidence that we are creating a great and unique lab activity from which students will benefit. Not merely performing stellar spectroscopy, but understanding the methods of obtaining, processing, analyzing, and interpreting data will deepen overall knowledge of the work involved in astronomy. After spending a great deal of time investigating RSpec's capabilities (of which we may have only scratched the surface) and working on the "how to's" of development, we are now ready to finalize the lab activity and implement a test run in the spring 2018 semester.

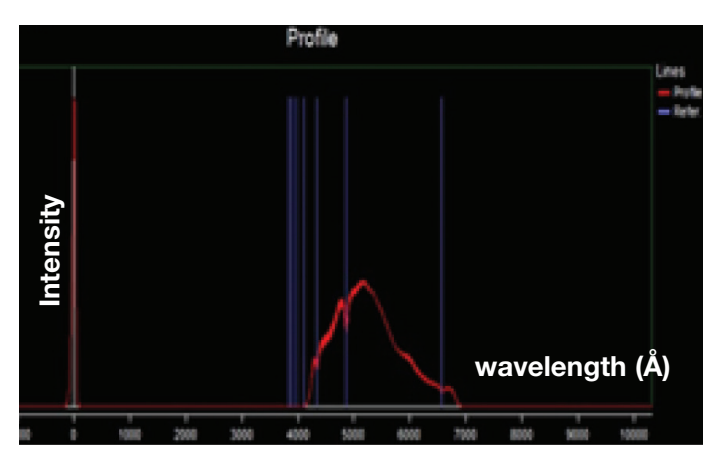

Fig. 5. Spectral analysis of Sirius using RSpec.

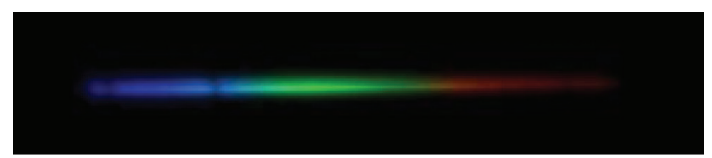

Fig. 6. Spectrum of Sirius.

\section{Authors' note}

If you are interested in investigating RSpec or Nebulosity, we have provided some information. Both packages do offer 30 -day trials. No compensation was given to the authors or institutions for use or mention of these products.

\section{RSpec:}

https://www.rspec-astro.com/

Cost $=\$ 109.00$

\section{Nebulosity:}

http://www.stark-labs.com/nebulosity.html

Cost $=\$ 95.00$

\section{Acknowledgments}

We would like to thank Tom Field, creator of RSpec, for being so supportive. We would also like to thank additional students who contributed: Mariah Birchard, Matthew Calvelo, Cameron Smith, and Hunter Stark. 